Identify (14) with (1) and apply the criterion (4). It then follows that (13) is the only solution of (14) satisfying (12) if the condition $F \leq 0$, which corresponds to (4), is satisfied. But the definition (16) shows that the condition $F \leq 0$ is identical with assumption (10). Since (12) is equivalent to (2), and (13) to (3), the proof is complete.

\title{
REFERENCES
}

1. L. Lichtenstein, Article II C 12 in the Enc. der Math. Wiss., vol. II. $3_{2}$, p. 1298 (1924)

2. E. Picard, Leçons sur quelques problèmes aux limites de la théorie des équations différentielles, Paris, 1930, p. 8

\section{A NOTE ON THE TRANSVERSE VIBRATION OF A TUBE CONTAINING FLOWING FLUID*}

BY GEORGE H. HANDELMAN† (Carnegie Institute of Technology)

1. Introduction. The problem of determining the lowest frequency of vibration of a tube containing flowing fluid has recently received considerable attention. Long $[1]^{1}$ has considered the tube as a beam and has calculated the frequencies for various end conditions by a power series method. He has pointed out that care must be taken in evaluating the resulting high order determinants to avoid erroneous results. Niordson [2] has given a very elegant treatment of the problem based on shell theory and has derived the beam equation as one approximation. Furthermore, for the case of simply supported ends, he has given closed analytic solutions for two limiting cases of the parameters and numerical results for other values. It is the purpose of this note to show that it is possible to determine the nature of the frequencies for the various end conditions used by Long for two ranges of the flow velocity solely from the structure of the differential equation without determining specific solutions.

Long has written the differential equation governing the deflection of the beam in the following non-dimensional form:

$$
F^{\mathrm{IV}}+k u^{2} F^{\mathrm{II}}+i u D F^{\prime}-D^{2} F=0
$$

where $F$ is the deflection and $u$ the flow velocity (both in non-dimensional terms). Roman numerals denote differentiation with respect to $x(0 \leqq x \leqq 1)$ and $D$ is the eigenvalue found by removing the time dependent term $\exp (i D t)$ from the original partial differential equation. The boundary conditions considered are
Both ends simply supported: $F(0)=F^{\prime \prime}(0)=F(1)=F^{\prime \prime}(1)=0$,
Both ends fixed:$$
F(0)=F^{\prime}(0)=F(1)=F^{\prime}(1)=0,
$$
Fixed-simple ends:$$
F(0)=F^{\prime}(0)=F(1)=F^{\prime \prime}(1)=0,
$$
Fixed-free ends:$$
F(0)=F^{\prime}(0)=F^{\prime \prime}(1)=F^{\prime \prime \prime}(1)=0 \text {. }
$$

${ }^{*}$ Received March 3, 1955. This research has been supported by the United States Air Force through the Office of Scientific Research of the Air Research and Development Command.

$\dagger$ Now at Rensselaer Polytechnic Institute.

${ }^{1}$ Numbers in square brackets refer to the list of references given at the end of this note. 
2. Small flow velocities. The behavior of the frequencies for small velocities of flow can be determined by the usual perturbation technique [3]. It should be observed that the differential equation (1) and boundary conditions specified do not form a selfadjoint problem; however, such will be the case for $u=0$.

Consider expansions of the form

$$
\begin{aligned}
& F=F_{0}(x)+u F_{1}(x)+u^{2} F_{2}(x)+\cdots \\
& D=D_{0}+u D_{1}+u^{2} D_{2}+\cdots .
\end{aligned}
$$

If these are inserted in Eq. (1) and the coefficients of each power of $u$ equated to zero, the following equations for the first three terms result:

$$
\begin{array}{ll}
F_{0}^{\mathrm{IV}}-D_{0}^{2} F_{0}=0 & \left(u^{0} \text { term }\right), \\
F_{1}^{\mathrm{IV}}-D_{0}^{2} F_{1}=2 D_{0} D_{1} F_{0}-i D_{0} F_{0}^{\prime} & \left(u^{1} \text { term }\right), \\
F_{2}^{\mathrm{IV}}-D_{0}^{2} F_{2}=-k F_{0}^{\prime \prime}+2 D_{0} D_{1} F_{1}+2 D_{0} D_{2} F_{0}-i D_{1} F_{0}^{\prime}-i D_{0} F_{1}^{\prime} & \left(u^{2} \text { term }\right) .
\end{array}
$$

Both sides of Eq. (3) are now multiplied by $F_{0}$ and the resulting expression is integrated from 0 to 1 . If this equation is now integrated by parts and account taken of the boundary conditions, it is found that for all cases

$$
\int_{0}^{1} F_{0}\left(F_{1}^{\mathrm{IV}}-D_{0}^{2} F_{1}\right) d x=\int_{0}^{1} F_{1}\left(F_{0}^{\mathrm{IV}}-D_{0}^{2} F_{0}\right) d x=2 D_{1} D_{0} \int_{0}^{1} F_{0}^{2} d x-\left.i D_{0} \frac{F_{0}^{2}}{2}\right|_{0} ^{1} .
$$

The left-hand side vanishes by virtue of Eq. (2). Furthermore, the first three sets of boundary conditions require that $F(0)=F(1)=0$. Hence, in these cases $D_{1}=0$. This result agrees with that found by Niordson in the simply supported case, for his computations show that if $D$ is plotted as a function of $u$, the curve has a horizontal tangent at $u=0$.

On the other hand, for the fixed-free end, $F_{0}(1) \neq 0$. Hence,

$$
D_{1}=\frac{i F_{0}^{2}(1)}{4 \int_{0}^{1} F_{0}^{2} d x}=i p,
$$

where $p>0$. Thus the time dependent term becomes

$$
\exp \left[i\left(D_{0}+u D_{1}\right) t\right]=\exp \left[i\left(D_{0}+i u p\right) t\right]=\exp \left(i D_{0} t\right) \exp (-u p t) .
$$

Hence damping takes place when terms through the first order are retained. This agrees with the calculations made by Long using polynomial expansions.

If one of the first three sets of boundary conditions holds (denoted as "supported end cases" in the following), the coefficient $D_{2}$ can be obtained by multiplying Eq. (4) by $F_{0}$, integrating from 0 to 1 , and noting that $D_{1}=0$. Again, after integration by parts, this equation becomes

$$
0=k \int_{0}^{1} F_{0}^{\prime 2} d x+2 D_{0} D_{2} \int_{0}^{1} F_{0}^{2} d x-i D_{0} \int_{0}^{1} F_{0} F_{1}^{\prime} d x .
$$

The term $\int_{0}^{1} F_{0} F_{1}^{\prime} d x$ can be examined by noting that if $F_{1}$ is written as the complex function $F_{1}=y+i v$, the real and imaginary parts satisfy

$$
\begin{aligned}
& y^{\mathrm{IV}}-D_{0}^{2} y=0, \\
& v^{\mathrm{IV}}-D_{0}^{2} v=-D_{0} F_{0}^{\prime},
\end{aligned}
$$


together with one of the sets of boundary conditions for supported ends given in Sec. 1 . Comparison with Eq. (2) then shows that $y=F_{0}$. Thus

$$
\begin{aligned}
\int_{0}^{1} F_{0} F_{1}^{\prime} d x & =\int_{0}^{1} F_{0} F_{0}^{\prime \prime} d x+i \int_{0}^{1} F_{0} v^{\prime} d x \\
& =\left.\frac{F_{0}^{2}}{2}\right|_{0} ^{1}+\left.i F_{0} v\right|_{0} ^{1}-i \int_{0}^{1} F_{0}^{\prime} v d x=-i \int_{0}^{1} F_{0}^{\prime} v d x .
\end{aligned}
$$

The sign of the last term can be determined by multiplying Eq. (6) by $v$ and integrating from 0 to 1 . Subsequent integration by parts yields

$$
-D_{0} \int_{0}^{1} F_{0}^{\prime} v d x=\int_{0}^{1} v\left(v^{\mathrm{IV}}-D_{0}^{2} v\right) d x=\int_{0}^{1}\left(v^{\prime 2}-D_{0}^{2} v^{2}\right) d x .
$$

Now the minimum principle corresponding to Eq. (2) states that for all functions $w$ satisfying suitable differentiability requirements and the boundary conditions, $D_{0}^{2}=\min$ $\int_{0}^{1} w^{\prime \prime 2} d x / \int_{0}^{1} w^{2} d x$. The function $v$ is such an admissible function and hence the righthand side of (7) is always greater than or equal to zero. Therefore, $-D_{0} \int_{0}^{1} F_{0}^{\prime} v d x x 0$. This remark and Eq. (5) then show that

$$
D_{2}=-\frac{k \int_{0}^{1} F_{0}^{\prime 2} d x+D_{0} \int_{0}^{1} F_{0}^{\prime} v d x}{2 D_{0} \int_{0}^{1} F_{0}^{2} d x} \leqq 0 .
$$

Thus for terms up to and including $u^{2}$, the frequency for the supported end cases decreases as $u$ increases and no damping occurs. This is in agreement with the numerical results previously obtained.

3. Velocities near the critical velocity. Niordson has observed in the simply supported case, that there is a critical velocity $u$ for which the frequency $D$ vanishes. The equation for this critical velocity is obtained by setting $D=0$ in Eq. (1). The resulting equation is just the ordinary column buckling equation with $k u^{2}$. playing the role of the buckling load. Niordson noted that the bar will be unstable for flow speeds greater than this critical velocity.

In similar fashion, it is seen that there will be a critical velocity for the other supported end cases which can be found from the corresponding buckling problems. On the other hand, there is no critical velocity for the fixed-free case since the differential equation $F^{\mathrm{IV}}+k u^{2} F^{\prime \prime}=0$ has only trivial solutions for these boundary conditions [4]. For this case then, there is no special velocity $u$ in the neighborhood of which a radical change in the analytic character of $D$ can occur.

The behavior of the frequency $D$ for velocities near the critical velocity in the supported end cases can be readily determined by a perturbation solution in terms of powers of $D$. If

$$
\begin{aligned}
& u=u_{0}+u_{1} D+u_{2} D^{2}+\cdots, \\
& F=F_{0}+F_{1} D+F_{2} D^{2}+\cdots,
\end{aligned}
$$

the equations for the first three terms obtained from Eq. (1) are

$$
\begin{aligned}
& F_{0}^{1 \mathrm{v}}+k u_{0}^{2} F_{0}^{\prime \prime}=0 \quad\left(D^{n}\right. \text { term), } \\
& F_{1}^{1 \mathrm{v}}+k u_{0}^{2} F_{1}^{\prime \prime}=-2 k u_{0} u_{1} F_{0}^{\prime \prime}-i u_{0} F_{0}^{\prime} \quad \text { (D'term), } \\
& F_{2}^{1 \mathrm{~V}}+k u_{0}^{2} F_{2}^{\prime \prime}=-2 k u_{0} u_{1} F_{1}^{\prime \prime}-k u_{1}^{2} F_{0}^{\prime \prime}-2 k u_{0} u_{2} F_{0}^{\prime \prime} \\
& -i u_{1} F_{0}^{\prime}-i u_{0} F_{1}^{\prime}+F_{0} \quad\left(D^{2} \text { term }\right) .
\end{aligned}
$$


The coefficient $u_{1}$ can be found by multiplying Eq. (9) by $F_{0}$ and integrating from 0 to 1 . Integration by parts yields

$$
0=-\left.2 k u_{0} u_{1} F_{0} F_{0}^{\prime}\right|_{0} ^{1}+2 k u_{0} u_{1} \int_{0}^{1} F_{0}^{\prime 2} d x-\left.i u_{0} \frac{F_{0}^{2}}{2}\right|_{0} ^{1} .
$$

The first and third terms vanish by virtue of the boundary conditions and therefore $u_{1}=0$. This result states that if $u$ is considered as a function of $D$ in the neighborhood of the critical velocity, the curve will have a horizontal tangent. Alternatively, if $D$ is considered as a function of $u$ (as in Sec. 2) in the same neighborhood, the curve will have a vertical tangent. This agrees with Niordson's results for the simply supported case. Furthermore, it indicates why an expansion in powers of $D$ is appropriate rather than one in powers of perturbations from the critical velocity.

The coefficient $u_{2}$ is determined by multiplying Eq. (10) by $F_{0}$, noting that $u_{1}=0$, and integrating from 0 to 1 . Partial integration leads to

$$
0=2 k u_{0} u_{2} \int_{0}^{1} F_{0}^{\prime 2} d x+\int_{0}^{1} F_{0}^{2} d x+i u_{0} \int_{0}^{1} F_{1} F_{0}^{\prime} d x,
$$

and the character of $u_{2}$ is then determined by examining the properties of $i u_{0} \int_{0}^{1} F_{1} F_{0}^{\prime} d x$. Let $F_{1}$ be written as $F_{1}=\xi+i \eta$. If Eq. (9) is then split into its real and imaginary parts

$$
\begin{aligned}
& \xi^{\mathrm{IV}}+k u_{0}^{2} \xi^{\prime \prime}=0, \\
& \eta^{\mathrm{IV}}+k u_{0}^{2} \eta^{\prime \prime}=-u_{0} F_{0}^{\prime} .
\end{aligned}
$$

Comparison of Eq. (11) with Eq. (8) shows that $\xi=F_{0}$; therefore,

$$
i u_{0} \int_{0}^{1} F_{1} F_{0}^{\prime} d x=\left.i u_{0} \frac{F_{0}^{2}}{2}\right|_{0} ^{1}-u_{0} \int_{0}^{1} \eta F_{0}^{\prime} d x=-u_{0} \int_{0}^{1} \eta F_{0}^{\prime} d x .
$$

Equation (12) can then be multiplied by $\eta$ and integrated from 0 to 1 to yield

$$
-u_{0} \int_{0}^{1} \eta F_{0}^{\prime} d x=\int_{0}^{1} \eta\left(\eta^{\mathrm{IV}}+k u_{0}^{2} \eta^{\prime \prime}\right) d x=\int_{0}^{1}\left(\eta^{\prime \prime 2}-k u_{0}^{2} \eta^{\prime 2}\right) d x
$$

The minimum principle associated with Eq. (8) states that for all functions $w$ satisfying suitable differentiability conditions and the boundary conditions, $k u_{0}^{2}=\min \int_{0}^{1} w^{\prime \prime 2} d x /$ $\int_{0}^{1} w^{\prime 2} d x$. The function $\eta$ is such an admissible function. Hence

and

$$
p=-u_{0} \int_{0}^{1} \eta F_{0}^{\prime} d x \geqq 0,
$$

$$
u_{2}=-\frac{\int_{0}^{1} F_{0}^{2} d x-p}{2 k u_{0} \int_{0}^{1} F_{0}^{\prime 2} d x}<0 .
$$

If terms through $D^{2}$ only are retained, the behavior in the neighborhood of the critical velocity $u_{0}$ is expressed by

$$
u-u_{0}=u_{2} D^{2}=-\left|u_{2}\right| D^{2} .
$$

If the flow velocity is less than the critical velocity, $u<u_{0}, D= \pm\left(\left|u-u_{0}\right| /\left|u_{2}\right|\right)^{1 / 2}$. Thus $D$ is real and there is neither damping nor exponential growth. On the other hand, if the flow velocity is greater than the critical velocity, $u>u_{0}, D= \pm i\left(\left|u-u_{0}\right| /\right.$ $\left.\left|u_{2}\right|\right)^{1 / 2}$. In this instance, $D$ is imaginary and the time dependent factor consists of an 
exponentially growing term as well as a damping term. With the exception of very special initial conditions, both terms will be present and the tube will be unstable.

4. Further remarks. It appears that the behavior of the higher order perturbation terms cannot be obtained as simply as those discussed above. These may require explicit determination of the function $F_{0}, F_{1}, F_{2}, \cdots$. It should be noted, however, that if $F_{0}, \cdots, F_{n-1}$ have been found, $D_{n}$ can be determined by quadratures. Furthermore, the differential equation for $F_{n}$ will be of the form

$$
F_{n}^{\mathrm{IV}}-D_{0}^{2} F_{n}=\text { previously determined functions. }
$$

The Green's function for this equation, in the case of simply supported ends is known [5], and can be determined for the other boundary conditions by standard methods. Thus $F_{n}$ can be found by integration.

These comments indicate that the perturbation terms can be computed step-by-step by quadratures. Furthermore, in the supported end cases the critical velocity can be determined beforehand and perturbation from this point in powers of $D$ will serve as a check on the perturbation solution in terms of powers of $u$.

\section{REFERENCES}

[1] R. H. Long, Jr., Experimental and theoretical study of transverse vibration of a tube containing flmving fluid, A.S.M.E., Preprint 54-A-22

[2] F. I. N. Niordson, Vibrations of a cylindrical tube containing flowing fluid, Trans. Roy. Inst. Tech., Stockholm, Nr. 73, (1953)

[3] Iothar Collatz, Eigenwertprobleme, Chelsea Publishing Co., New York, 1948, p. 305 ff.

[4] I. Collatz, Ibid, p. 54

[5] I. Collatz, Ibid, p. 84

\section{ON AN OSCILLATION CRITERION OF DE LA VALLEE POUSSIN*}

\section{By PHILIP HARTMAN AND AUREL WINTNER (The Johns Hopkins University)}

An oscillation criterion of de la Vallée Poussin ${ }^{1}$ on homogeneous, linear differential equations of order $n$, when particularized ${ }^{2}$ to $n=2$, runs as follows: Let both coefficient functions of

$$
x^{\prime \prime}+g(t) x^{\prime}+f(t) x=0
$$

be real-valued and continuous on a $t$-interval and suppose that (1) has a solution $x(t) \not \equiv 0$ which vanishes for at least two points of that $t$-interval, say at $t=0$ and at $t=h>0$ (so that

$$
x(0)=0, \quad x(h)=0,
$$

where, without loss of generality, $x(t) \neq 0$ when $0<t<h)$. Then

$$
1<M_{1} h+M_{2} h^{2} / 2,
$$

${ }^{*}$ Received March 10, 1955.

'G. Sansone, Equazioni differenziali nel campo reale, vol. 1, 1948, p. 183.

2F. Tricomi, Equazioni differenziali, 1948, p. 110. 